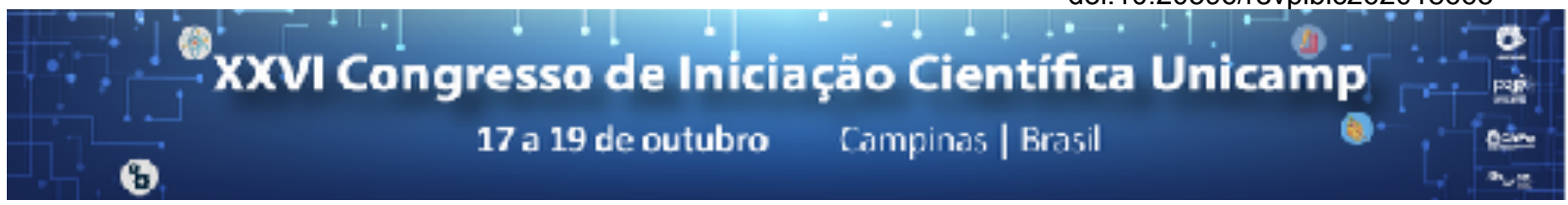

\title{
Avaliação da dor pós-operatória após o tratamento endodôntico com ampliação foraminal executada por alunos de especialização
}

\author{
Jéssica Jeuken Teixeira*, José Flávio A. de Almeida
}

\section{Resumo}

O controle de dor pós-operatória é de extrema importância, fazendo parte do tratamento ao paciente. Esse levantamento avaliou a dor pós-operatória após o tratamento endodôntico com ampliação foraminal em sessão única ou múltipla realizada por alunos de especialização na faculdade de Odontologia de Piracicaba, 24 horas após a conclusão do tratamento (dente obturado).

\section{Palavras-chave:}

Dor pós-operatoria, endodontia, forame apical

\section{Introdução}

O controle da dor pós-operatória é de extrema importância, fazendo ainda parte do tratamento ao paciente. Considerando isso, o objetivo do presente estudo foi avaliar a incidência de dor pós-operatória gerada após tratamento endodôntico com ampliação foraminal em sessão única ou múltipla, realizado por alunos do curso de especialização em endodontia na Faculdade de Odontologia de Piracicaba.

\section{Resultados e Discussão}

Os resultados obtidos na escala de dor verbal numérica foram variados no período pós-operatório. Trinta e quatro por cento dos pacientes apresentava sintomatologia anteriormente ao tratamento realizado. Esses pacientes que apresentavam sintomatologia prévia ao tratamento, demonstraram maior índice de dor no período pós-operatório, quando comparados com os que não apresentavam sintomatologia prévia.

Da mesma forma que observada relação entre dor pré e pós-operatória, o número de medicações utilizadas pelos pacientes submetidos aos tratamento com presença de dor pré-operatória também foi significantemente maior do que nos casos de ausência de dor pré-operatória.

Trinta e quatro por cento dos pacientes apresentaram dor pós-operatória independentemente do diagnóstico pulpar e periapical encontrados clinicamente. Dessa forma, a maior parte dos pacientes não apresentou dor após o tratamento e não foi possível encontrar diferença estatisticamente significante quando relacionados os dados da escala de dor verbal numérica e os diferentes diagnósticos clínicos.

A análise do número de medicações tomadas foi diferente para os diagnósticos pulpar e periapical. Os pacientes que foram submetidos ao tratamento de pulpite ingeriram mais medicações que os pacientes que já tinham a $1^{\text {a }}$ fase feita ou necrose pulpar. As demais comparações no diagnóstico pulpar não diferiram. Para o diagnóstico periapical, não foi encontrada diferença estatisticamente significante do número de medicações tomadas no período pós-operatório.
Quando comparado o número de medicações tomadas e os diferentes testes clínicos realizados, o resultado se comportou de maneira semelhante. O número de medicações ingeridas pelos pacientes que apresentaram teste de sensibilidade positivo foi maior que os que apresentaram teste de sensibilidade negativo. Da mesma forma, para os testes de percussão e palpação não foram encontradas diferenças entre os resultados.

Os tratamentos endodônticos foram finalizados em uma ou duas sessões. O número de casos concluídos em duas sessões foi pequeno $(n=9)$ quando comparado com os concluídos em sessão única $(n=91)$. Não houve diferença na incidência de dor pós-operatória para casos tratados em uma ou duas sessões. Entretanto, os pacientes tratados em duas sessões ingeriram mais medicações dos que os tratados em sessão única.

\section{Conclusões}

1 - A maioria dos pacientes que passaram por tratamento endodôntico com ampliação foraminal não apresentou sintomatologia pós-operatória.

2 - A presença de dor pré-operatória é um fator que influencia a dor pós-tratamento mesmo em casos tratados com instrumentação além do ápice.

3 - Com ampliação foraminal, casos de pulpite tratados endodonticamente apresentaram maior incidência de dor pós-operatório que casos necrosados.

1.Alsomadi L, Al Habahbeh R. Role of Prophylactic Antibiotics in the Management of Postoperative Endodontic Pain. J Contemp Dent Pract. 2015

2.Attar S, Bowles WR, Baisden MK, et al. Evaluation of pretreatment analgesia and endodontic treatment for postoperative endodontic pain. J Endod 2008;34:652-5. 14

3.Bergmans L, Van Cleynenbreugel J, Wevers M, Lambrechts P. Mechanical root canal preparation with NiTi rotary instruments: rationale, performance and safety Status report for the American Journal of Dentistry. Am J Dent. 2001 Oct;14(5): 324-33.

4.DiRenzo A, Gresla T., Bradford R.J., Rogers M., Tucker D., BeGole E.A. Postoperative pain after 1- and 2-visit root canal therapy. Oral Surg Oral Med Oral Pathol Oral Radiol Endod 2002; 93:605-10. 